\title{
Pattern electroretinogram and peripheral colour contrast thresholds in ocular hypertension and glaucoma: comparison and correlation of results
}

\author{
Simon T Ruben, Geoffrey B Arden, Fiona O'Sullivan, Roger A Hitchings
}

\begin{abstract}
Aims-Both pattern electroretinogram and peripheral colour contrast thresholds have been shown to be abnormal in glaucoma and ocular hypertension. This study evaluates each of these tests as tools for the early diagnosis of glaucoma, compares and contrasts the results, and examines the relation between the two tests in a large cohort of ocular hypertensive patients.

Methods-Transient and steady state pattern electroretinograms and peripheral colour contrast thresholds were performed in 45 normal, 37 glaucomatous, and 206 ocular hypertensive eyes. The results were analysed using receiver operating characteristic curves, together with evaluation of sensitivity and specificity of the tests. The relation between the two tests was examined by direct statistical correlation of the results.

Results-All tests showed high sensitivity and specificity for discriminating between normal and glaucomatous eyes. However, there was a significant difference between the two tests for the number of ocular hypertensives considered as abnormal. Forty per cent of ocular hypertensives had abnormal pattern electroretinogram compared with $30 \%$ with abnormal peripheral colour vision. Peripheral colour contrast thresholds showed a significant correlation with both transient and steady state pattern electroretinogram.

Conclusion-Both of these tests have been shown to be promising new tools for the early detection of glaucoma but the number of ocular hypertensive patients showing abnormal results is rather higher than expected considering the natural history of the condition. Sensitivity in ocular hypertension may be increased by using a combination of both tests. The significant correlation between these psychophysical and electrophysiological tests is discussed.
\end{abstract}

(Br f Ophthalmol 1995; 79: 326-331)

There has been increasing interest in the use of electrophysiological and psychophysical tests as diagnostic tools for early glaucomatous visual loss during the past 10 years. ${ }^{1-3}$ This interest has been fuelled by the finding that a significant proportion of ganglion cells may be lost before a focal field defect becomes apparent on either manual or automated perimetry. ${ }^{4}$ Attention has thus been focused on electrophysiological techniques such as the pattern electroretinogram, which has been shown to be a sensitive detector of optic nerve disfunction in both glaucoma and other optic neuropathies. ${ }^{6-8}$ Psychophysical techniques have also been studied extensively. Both short wavelength perimetry ${ }^{9-11}$ and motion detection techniques ${ }^{12-15}$ are of particular interest as they provide non-invasive, easily performed tests of visual function that may be more sensitive at detecting early glaucomatous optic nerve damage than present techniques. Other psychophysical tests that have been investigated include central ${ }^{16}$ and peripheral colour contrast thresholds, ${ }^{17}$ and flicker sensitivity. ${ }^{18}$

The use of pattern electroretinogram (PERG) ${ }^{619}$ and colour contrast thresholds ${ }^{17}$ to discriminate between normal and glaucomatous eyes have both been investigated in this department and early results reported. In addition, both tests have been shown to have significantly reduced results in a proportion of ocular hypertensive patients and may have a role in the management of such patients. The long term longitudinal studies needed to establish the true value of these tests over more conventional field analysers in the diagnosis of glaucoma are under way. The purpose of this study is to compare the results of an electrophysiological test (PERG) with those of a psychophysical test (peripheral colour contrast) in normal, glaucomatous, and ocular hypertensive eyes. We investigated the sensitivity and specificity of the two tests and the correlation between them in a large group of ocular hypertensive patients.

Patients, materials, and methods

Thirty seven glaucomatous, 206 ocular hypertensive, and 45 normal eyes were studied. All patients recruited form part of an ongoing longitudinal study of psychophysical and electrophysiological tests and gave informed consent to participate. All patients had PERG and peripheral colour contrast tests in addition to Humphrey automated visual field analysis (program 24-2) at the same visit. The tests were carried out in random order so as not to introduce any bias that may be caused by, for example, fatigue. Full clinical examination, including measurement of intraocular pressure and gonioscopy, was carried out following the psychophysical and electrophysiological testing.

Ocular hypertensive patients all had IOP in excess of $24 \mathrm{~mm} \mathrm{Hg}$ (mean of three 
Table 1 Results of PERG and peripheral colour contrast thresholds for normal, ocular hypertensive (OHT), and glaucomatous eyes. Mean (SD)

\begin{tabular}{|c|c|c|c|}
\hline Variable & $\begin{array}{l}\text { Normals } \\
(n=60)\end{array}$ & $\begin{array}{l}O H T \\
(n=206)\end{array}$ & $\begin{array}{l}\text { Glaucoma } \\
(n=37)\end{array}$ \\
\hline $\begin{array}{l}\text { Age (years) } \\
\text { P50 }(\mu \mathrm{V}) \\
\text { N95 }(\mu \mathrm{V}) \\
\text { P50+N95 ( } \mu \text { V) } \\
\text { Steady state PERG }(\mu \mathrm{V}) \\
\text { Tritan threshold }(\%) \\
\text { Protan threshold }(\%) \\
\text { Deuteran threshold }(\%)\end{array}$ & $\begin{array}{l}59(14) \\
2.54(0.59) \\
0.96(0.41) \\
3.5(0.7) \\
2.4(0.53) \\
18.4(8.9) \\
18.5(6.4) \\
19.5(8.3)\end{array}$ & $\begin{array}{l}60(11) \\
1.95(0 \cdot 8)^{\star} \\
1.12(0.8)^{\star} \\
3.07(1.3)^{\star} \\
2.02(0.87)^{\star} \\
21.88(10 \cdot 8)^{\star} \\
22.8(37 \cdot 7) \\
22.6(9.8)\end{array}$ & $\begin{array}{l}64(7) \\
1.38(0.52) \dagger \ddagger \\
0.61(0.51) \dagger \ddagger \\
1.99(0.8) \dagger \ddagger \\
1.45(0.48) \dagger \ddagger \\
44.6(27 \cdot 2) \dagger \ddagger \\
38.9(25 \cdot 6) \dagger \ddagger \\
41.7(24.8) \dagger \neq\end{array}$ \\
\hline
\end{tabular}

Two sample $t$ test: ${ }^{\star}$ OHT compared with normals: $\mathrm{p}<0 \cdot 05$; †OHT compared with glaucoma: $\mathrm{p}<0.002$; $¥$ Normals compared with glaucoma: $\mathrm{p}<0 \cdot 0001$.

consecutive recordings). Normal Humphrey visual fields were defined as no point of the 24-2 program having a sensitivity reduced by greater than $5 \mathrm{~dB}$ compared with age matched controls. None of the ocular hypertensive patients were on any form of pressure lowering treatment at the time of testing. One eye of each subject was chosen at random for the analysis of results.

The glaucoma patients had an IOP of $21 \mathrm{~mm} \mathrm{Hg}$ or below either on topical $\beta$ blocker medication or having undergone filtration surgery. Patients were diagnosed as having glaucoma on the basis of a repeatable field defect on Humphrey automated perimetry (defined as at least three points depressed by greater than $5 \mathrm{~dB}$ compared with age matched controls) together with optic nerve changes consistent with glaucomatous damage. All glaucoma patients included had IOP greater
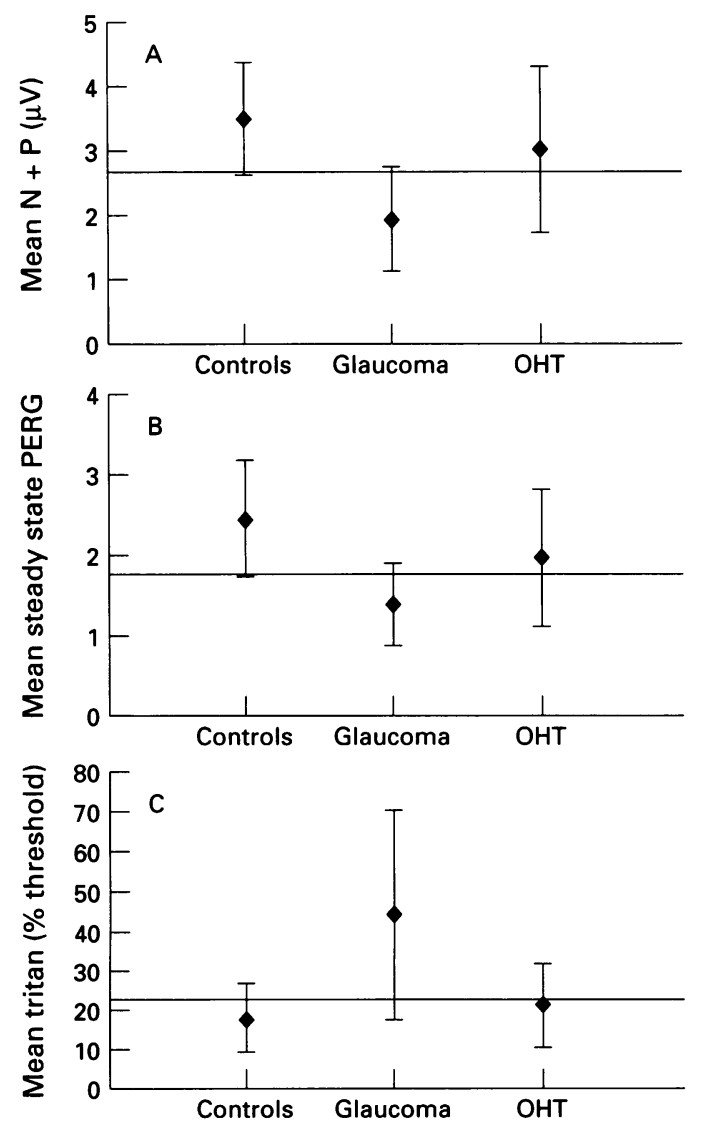

Figure 1 (A) Transient, (B) steady state PERGs, and (C) tritan thresholds in normal, ocular hypertensive, and glaucomatous eyes (means (SD)). Measurements of $N+P$ were made from the baseline and for steady state from peak were made from than $21 \mathrm{~mm} \mathrm{Hg}$ at the time of diagnosis (there were no low tension glaucoma patients).

Persons with normal eyes consisted of patients' spouses and hospital staff. Glaucoma patients with all stages of disease were included and were recruited from the glaucoma clinics of Moorfields Eye Hospital.

Subjects with other eye disease or systemic diseases including hypertension treated with systemic $\beta$ blockers and diabetes were excluded. All patients had corrected visual acuity of at least $6 / 9$ in the eye under investigation. One eye of each individual was selected at random for statistical analysis (except in patients with unilateral disease).

Electrophysiological and psychophysical tests were undertaken by experienced technicians without knowledge of the clinical status of the patients.

\section{PATTERN ELECTRORETINOGRAM}

The method used for PERG measurement has been described in previous reports. ${ }^{619}$ The PERG was recorded using gold foil corneal electrodes and a reference electrode on the skin near the lateral canthus. The stimulus subtended $16 \times 22^{\circ}$ and the pattern reversing checks were of $30^{\circ}$ subtense and near $100 \%$ contrast. Mean illumination was $100 \mathrm{~cd} / \mathrm{m}^{2}$. Transient responses measured at a pattern reversal frequency of 6 reversals per second and steady state responses measured at a pattern reversal frequency of 16 reversals per second were recorded for each eye. About 250 sweeps were averaged for each response and at least four responses were recorded for each eye. For the transient response, the initial corneal positive response (P50) and the later corneal negative response (N95) were measured separately.

PERIPHERAL COLOUR CONTRAST THRESHOLDS The method of measuring peripheral colour contrast has been described in detail in a previous publication. ${ }^{17}$ The equipment consists of a high resolution colour monitor driven by a computer with special graphics card and software. The image on the screen was a central white fixation spot, a uniform background of $16 \mathrm{~cd} / \mathrm{m}^{2}$, and a colour contrast annulus concentric with the fixation spot. Annulus and background were of the same luminance and this varied slightly depending on the subject's flicker matches. The distance between screen and cornea was fixed at $45 \mathrm{~cm}$. The annulus had a radius of $12.5^{\circ}$ in the extramacular field and a width of $1^{\circ}$ of arc.

The test involved the removal of a $45^{\circ}$ segment of the annulus in one of four quadrants: right or left upper, right or left lower. The patient had to identify the correct quadrant. Threshold colour contrast was obtained with a modified binary search technique previously described. What was measured amounted to an average colour contrast threshold in the entire annular zone of the retinal image of the stimulus.

Peripheral colour contrast thresholds were 
Table 2 Specificity and sensitivity for different values of peripheral colour contrast threshold in normal and glaucomatous eyes (tritan axis)

\begin{tabular}{lllll}
\hline & \multicolumn{4}{l}{ Peripheral colour vision } \\
\cline { 2 - 5 } & 20 & 22 & 23 & 24 \\
\hline Specificity (\%) & 70 & 82 & 82 & 87 \\
Sensitivity (\%) & 89 & 86 & 86 & 78 \\
\hline
\end{tabular}

determined for tritan, deuteran, and protan axes.

\section{Results}

The patient characteristics and results of PERG and colour threshold tests are shown in Table 1 and Figure 1.

The PERG values for ocular hypertensives differ significantly from the normal values and these mirror previous reports from this department. ${ }^{619}$ Of the three colour contrast axes examined only the tritan axis showed a significant difference from the normal group which is in agreement with earlier studies. ${ }^{17}$ Because of this finding, only the tritan axis has been used
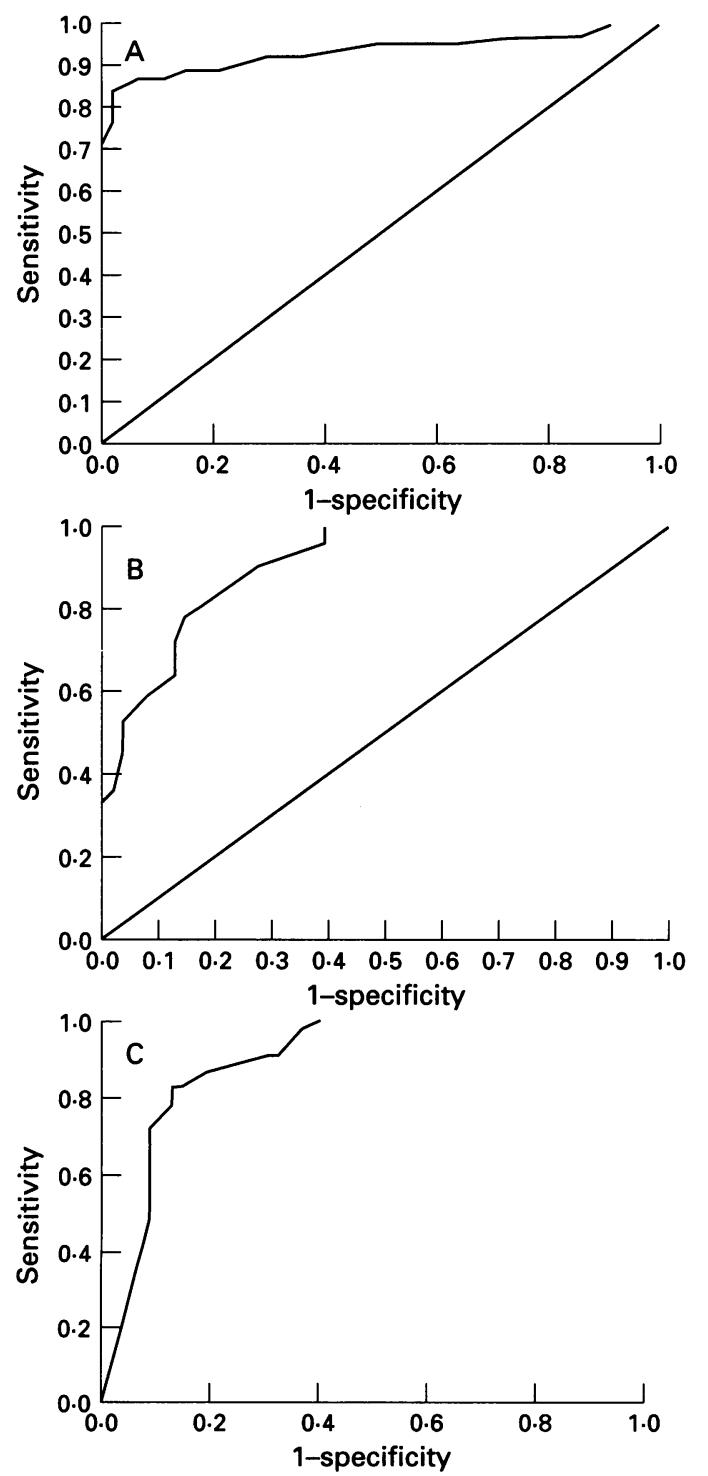

Figure 2 Receiver operating characteristic plots for (A) transient pattern electroretinogram, (B) steady state pattern electroretinogram, and (C) peripheral colour contrast thresholds.
Table 3 Proportion of ocular hypertensives considered to be abnormal on each test. (Normal values: tritan threshold $\leqslant 23 \%$, transient PERG $\geqslant 2 \cdot 7 \mu \mathrm{V}$, steady state PERG $\geqslant 1.8 \mu \mathrm{V})$

\begin{tabular}{lll}
\hline Variable & 'Abnormal' & 'Normal' \\
\hline Tritan threshold & $62(30 \%)$ & $144(70 \%)$ \\
Transient PERG & $83(40 \%)$ & $123(60 \%)$ \\
Steady state PERG & $84(41 \%)$ & $122(59 \%)$ \\
\hline
\end{tabular}

in subsequent analysis. The PERG values and all three peripheral colour contrast thresholds were significantly different from both the normal and ocular hypertensive eyes.

\section{PATTERN ELECTRORETINOGRAM}

Results for transient and steady state PERG are summarised in Figure $1 \mathrm{~A}$ and $\mathrm{B}$ and Table 1 . A value of $2 \cdot 7 \mu \mathrm{V}$ for the combined (P50+N95) amplitude of the transient PERG and $1.8 \mu \mathrm{V}$ for the steady state amplitude were found to have a high degree of sensitivity and specificity for discrimination between normal and glaucomatous eyes. Using this criterion specificity and sensitivity were both $90 \%$ for the transient PERG and $74 \%$ and $92 \%$ for the steady state PERG. Diagnostic precision (the percentage of eyes correctly diagnosed out of all normals and glaucomas) was $90 \%$ for the transient PERG and $85 \%$ for the steady state PERG.

Using these criteria for the lower limit of normality about $40 \%$ of the ocular hypertensive eyes would be considered abnormal (see below).

PERIPHERAL COLOUR CONTRAST

The peripheral colour contrast thresholds are summarised in Table 1 and Figure 1C. The glaucomatous eyes show significantly raised thresholds with ocular hypertensive eyes giving intermediate values, being the mean of a group containing normal and abnormal results. In these groups of normal and glaucomatous eyes there is some overlap of results from the two groups but similar changes are seen in each of the three colour axes measured. In a previous series $^{17}$ a high degree of separation between normals and glaucomatous eyes was found. Although specificity and sensitivity are high in this present series ( $82 \%$ and $86 \%$ respectively), there is some overlap between normal and glaucomatous eyes (see ROC plots below), depending on the value taken as being the upper limit of normal. A value between $22 \%$ and $24 \%$ for the tritan axis gives the best separation between normal and glaucomatous eyes for this series of patients (Table 2).

Sensitivity and specificity for both tests is high when comparing normal eyes with eyes with established glaucomatous visual loss. What is more important is the ability of the tests to diagnose early glaucomatous damage in patients with ocular hypertension.

RECEIVER OPERATING CHARACTERISTIC PLOTS To further illustrate the ability of each test to discriminate between normal and glaucoma, 
Table 4 Proportion of ocular hypertensives considered abnormal using more than one test (normal values as for Table $3(n=206)$ )

\begin{tabular}{lllll}
\hline \multicolumn{5}{l}{ PERG versus colour contrast } \\
\cline { 2 - 5 } & $\begin{array}{l}\text { Both } \\
\text { 'normal' }\end{array}$ & $\begin{array}{l}\text { 'Normal' PERG } \\
\text { with 'abnormal' } \\
\text { colour contrast }\end{array}$ & $\begin{array}{l}\text { 'Abnormal' PERG } \\
\text { with 'normal' } \\
\text { colour contrast }\end{array}$ & $\begin{array}{l}\text { Both } \\
\text { 'abnormal' }\end{array}$ \\
\hline Transient PERG & $96(47 \%)$ & $26(13 \%)$ & $48(23 \%)$ & $36(17 \%)$ \\
Steady state PERG & $92(44 \%)$ & $28(14 \%)$ & $53(26 \%)$ & $33(16 \%)$ \\
\hline
\end{tabular}

receiver operating characteristic (ROC) curves have been constructed for transient and steady state PERG and peripheral colour contrast thresholds (Fig 2A-C). The ROC plot is obtained by calculating the sensitivity and specificity for every possible value of a given variable and plotting sensitivity against 1specificity. A test that perfectly discriminates between the two groups would coincide with the left and top sides of the plot. The diagnostic precision as described above coincides with the area under the curve. ${ }^{20}$

It can be seen that the transient PERG gives the best discrimination between the
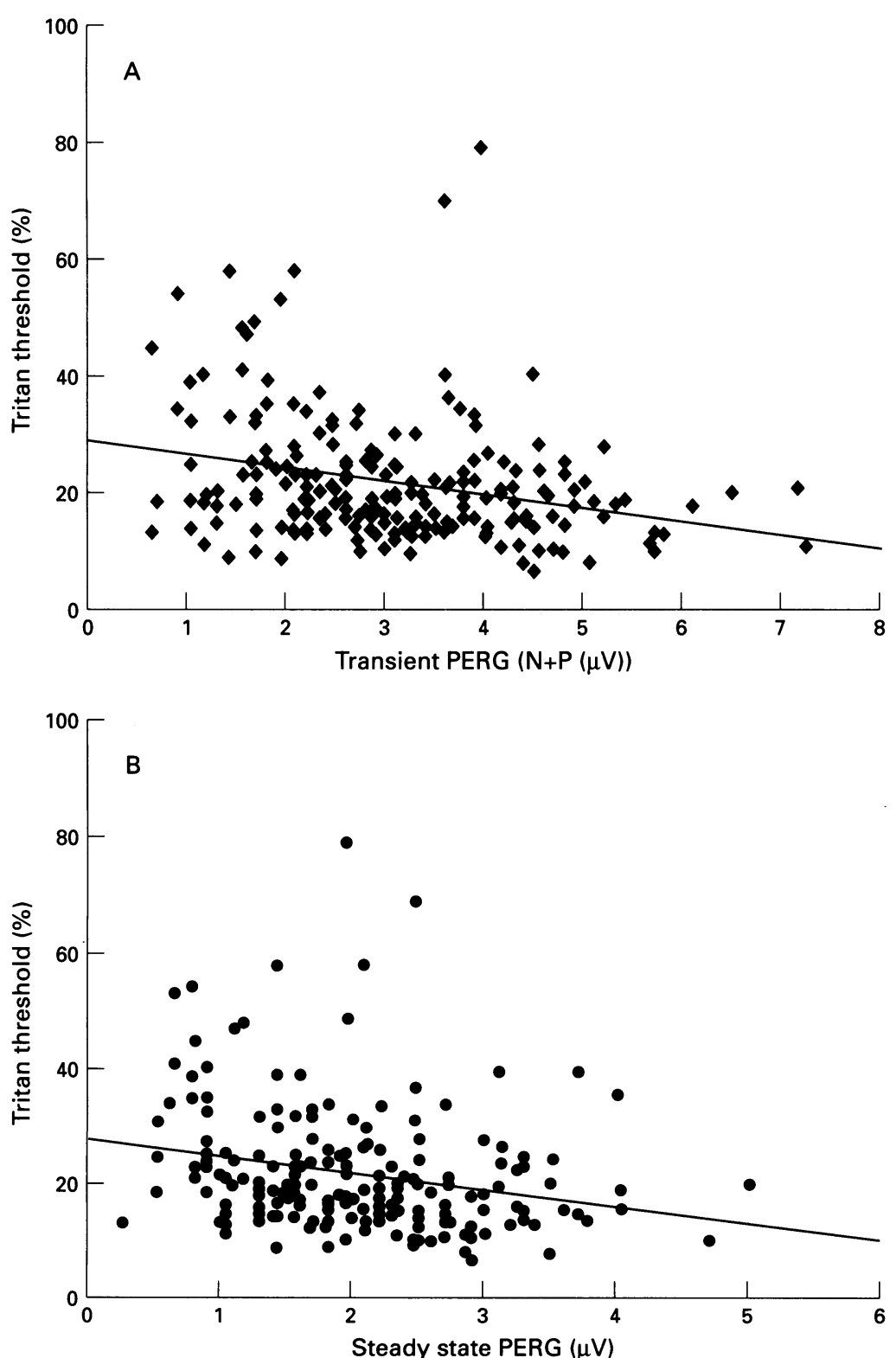

Figure 3 Scattergram plots and regression lines of peripheral colour contrast (tritan axis) against (A) transient PERG, (B) steady state PERG.
Table 5 Correlation coefficients and significance values for colour contrast thresholds compared with PERG measurements in normal, glaucomatous, and ocular hypertensive eyes

\begin{tabular}{llc}
\hline & $\mathrm{r}$ & $p$ Value \\
\hline Ocular hypertension: & -0.35 & $<0.0001$ \\
P50 & -0.075 & 0.14 \\
N95 & -0.27 & 0.0001 \\
N+P & -0.22 & 0.001 \\
Steady state & -0.45 & $<0.007$ \\
Normals: & -0.23 & 0.18 \\
P50+N95 & -0.09 & $>0.5$ \\
Steady state & -0.22 & $>0.2$ \\
Glaucoma: & & \\
P50+N95 & & \\
Steady state & & \\
\hline
\end{tabular}

two groups with the steady state and colour contrast thresholds having very similar curves.

RESULTS IN OCULAR HYPERTENSION

Using the results obtained in the normal and glaucoma groups it is possible to divide individual ocular hypertensive patients into those who have normal or abnormal results. Table 3 shows the proportion of the population of 206 ocular hypertensives who gave abnormal results for each test.

A significantly smaller number of the cohort are abnormal with the peripheral colour contrast test when compared with either PERG parameter: $\mathrm{p}<0.05$ using the $\chi^{2}$ test.

If a combination of PERG and peripheral colour contrast tests are used to evaluate normality versus abnormality in ocular hypertension, a higher degree of discrimination and therefore higher sensitivity can be obtained (Table 4).

COMPARISON OF PERG AND PERIPHERAL

COLOUR VISION

For the comparison of peripheral colour contrast thresholds with PERG (in ocular hypertensive eyes), only the tritan threshold was used. When the results from the ocular hypertensive group are plotted there is a significant correlation between peripheral colour contrast thresholds and both transient and steady state PERG (Fig 3A, B). Pearson's correlation: (i) for transient PERG; $r=-0.27$ $\mathrm{p}<0.0001$; (ii) for steady state PERG; $r=-0 \cdot 23, p=0 \cdot 001$. Neither the deuteran nor the protan axes showed any significant correlation with any PERG parameters.

Results for each PERG parameter (P50, $\mathrm{N} 95, \mathrm{~N}+\mathrm{P}$, and steady state) correlated with peripheral colour contrast thresholds (tritan axis) are shown in Table 5.

It can be seen that there is a weak but significant correlation between peripheral colour thresholds and both transient and steady state PERG amplitudes. However, the strongest relation is between the P50 component of the PERG and tritan values. Of the three colour axes measured only the tritan showed any significant correlation with the PERG results. Figure 4 shows the lack of a significant correlation between deuteran thresholds and the P50 PERG. Deuteran and protan thresholds gave 


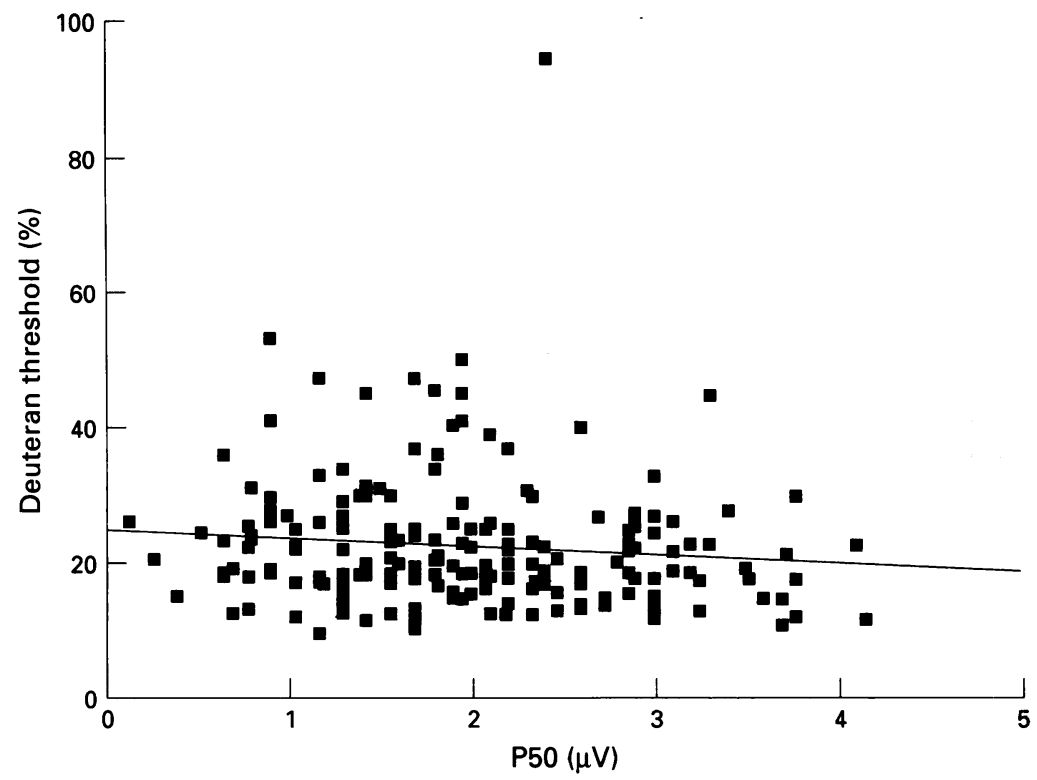

Figure 4 Scattergram plot of deuteran threshold against P50 PERG showing no significant correlation. have abnormal PERG results (about $40 \%$ ) than would be expected from the conversion rate of these patients to glaucoma which has been found to be nearer $10 \%$ in longitudinal follow up studies of ocular hypertensive patients not on treatment. ${ }^{22-24}$ The proportion of ocular hypertensives with abnormally high peripheral colour vision thresholds $(30 \%)$ is also on the high side. However, if one looks at the number of patients with abnormal results on both tests the value of $17 \%$ is more akin to the number of patients one would be expecting to develop glaucoma over a prolonged period. By using both tests, selectivity for diagnosing early glaucomatous damage may be increased. This is supported by the high specificity and sensitivity when using both tests together to discriminate between normals and established glaucoma. Obviously longitudinal studies of those patients converting to glaucoma will reveal the true value of these and other tests in the early diagnosis of glaucoma. The value of each test would also be dependent on the clinical setting in which they were to be used. For example, a high sensitivity would be acceptable as a screening test where the higher proportion of false positives could be eliminated by a second test. In this respect these tests would prove to be as effective as visual field testing and better than intraocular pressure measurement.

It can be seen from the ROC plots that the transient PERG is a little better at discriminating between normals and glaucomatous eyes than the steady state response. However, this difference must be looked at in light of the fact that the steady state response takes a considerably shorter time to perform and is much better tolerated by the patient. Thus in a screening situation the steady state response may be the better test with the option of performing the transient response for those who are abnormal.

Correlation coefficients are not the most powerful statistical method for analysing these results because of the wide variation of values in each group and the fact that the ocular hypertensive group probably includes two subpopulations: those with normal function and those with early optic nerve dysfunction. This simple analysis shows that there is a systematic variation between the members of the cohort, that it appears to be disease related, and that there is no good evidence that the two tests are analysing losses of different functions. However, in view of the spread of results, more sophisticated analyses were not attempted.

Furthermore, because of the differences between the retinal areas tested and the levels in the retina or central nervous system involved in producing the responses one could not expect to find a perfect correlation between the results of a psychophysical test and an electrophysiological test, even when used to discriminate between normal and severely damaged eyes as in glaucoma.

The correlation between the PERG and peripheral colour vision for the tritan axis must be interpreted in this light. The proportions of ocular hypertensives considered abnormal on this series the subjects were selected purely on their clinical state and not on their willingness to perform the tests.

As has been found previously, ${ }^{3}$ a higher proportion of ocular hypertensives were found to 
each test are significantly different. This is reflected to some degree by the low value of the correlation coefficient. The fact that a significant correlation is found can only support the hypothesis that both tests are measuring the same pathological process to some degree. The finding that only the tritan axis shows significant correlation with the PERG results and was also the only colour axis to be significantly reduced in ocular hypertension lends support to the theory of selective damage to the blue cone system early in the disease process, but that in more advanced glaucoma there is a disturbance of all retinal elements. On the other hand, a far from perfect correlation would be expected if there were more than one pathological process occurring in the early stages of glaucoma with perhaps different retinal elements affected to differing degrees.

Also of interest was the difference between the different variables of the PERG. The N95 component did not show a significant correlation with colour vision whereas the strongest correlation was with the P50 component. Steady state amplitudes which depend to a greater extent on the $\mathrm{N} 95$ component $^{25}$ also showed a significant correlation. Current source density analysis of the PERG suggests that the P50 component originates in the inner retina, whereas the N95 component is associated with ganglion cell or optic nerve activity. ${ }^{25}$ If in glaucoma the earliest changes were in the ganglion cell layer as histological changes have shown, ${ }^{45}$ we would expect early loss of the N95 component of the PERG and for this to correlate with other tests of early glaucoma. One explanation for our findings is that there is also early loss of function in the retina - possibly the photoreceptors - and this is reflected by changes in the colour contrast test and also a decrease of the P50 component of the PERG. Histological studies have shown loss of photoreceptors in eyes with secondary angle closure glaucoma and this may indicate that glaucoma may be associated with a loss of photoreceptors. ${ }^{26}$ Decreased sensitivity of the blue cone pathway has been utilised in blue-yellow perimetry which has also been advocated as a more sensitive measure of early glaucomatous damage. ${ }^{9-11}$

Another explanation could be that there is more than one type of defect occurring in early glaucoma and that both inner and outer retinal elements are affected to some degree at different stages of the disease or in different patient populations. Both P50 and N95 components of the PERG have been shown to be affected in patients with glaucoma. ${ }^{3}$ Outer retinal damage may occur later in the process which would explain the smaller number of ocular hypertensives giving abnormal results for peripheral colour vision.

Further studies are under way in order to establish the predictive values of these two tests. If more than one pathomechanism is involved in the early stages of glaucoma, a combination of tests that reflect differing aspects of visual function may be utilised to increase the sensitivity of diagnosing early damage in patients at risk of developing glaucoma.

The authors would like to thank the staff of the electrodiagnostic department for technical assistance. STR is supported by grant from the Guide Dogs for the Blind Association.

1 Korth $M$, Koca $M$. Clinical electrophysiology relevant for early glaucoma diagnosis. Curr Opin Ophthalmol 1993; 4: 22-8

2 Arden GB. Comparison of new psychophysics and perimetry with electrophysiological techniques in the diagnosis of glaucoma. Curr Opin Ophthalmol 1993; 4: 14-21.

3 Trick GL. The pattern electroretinogram in glaucoma and ocular hypertension. In: Heckenlively J, Arden GB, eds. Principles and practice of clinical electrophysiology of vision. Principles and practice of clinical

4 Quigley HA, Addicks EM, Green WR. Optic nerve damage in human glaucoma. III. Quantitative correlation of nerve fiber loss and visual field defect in glaucoma, ischaemic neuropathy, papilloedema, and toxic neuropathy. Arch Ophthalmol 1982; 100: 135-46.

5 Quigley HA, Dunkelberger BS, Green WR. Retinal ganglion cell atrophy correlated with automated perimetry in human eyes with glaucoma. Am f Ophthalmol 1989; 107: 453-64.

6 O'Donaghue E, Arden GB, O'Sullivan F, Falcao-Reis F, Moriarty B, Hitchings RA, et al. The pattern electroretinogram in glaucoma and ocular hypertension. $B r \mathcal{F}$ Ophthalmol 1992; 76: 387-94.

7 May JG, Ralston JV, Reed JL, van Dyk HJL. Loss in pattern elicited electroretinograms in optic nerve dysfunction. Science 1984; 211: 953-5.

8 Ringens PJ, Viifvinkel-Bruinenga S, Van Lith GHM. The pattern elicited electroretinogram. A tool in the early detection of glaucoma. Ophthalmologica 1986; 192: 171-5.

9 Sample PA, Weinreb RN, Progressive colour visual field loss in glaucoma. Invest Ophthalmol Vis Sci 1992; 33: 2068-71.

10 Johnson CA, Casson EJ, Shapiro LR. Progression of glaucomatous visual fieldloss over five years: a comparison of white-on-white and blue-on-yellow perimetry. Invest Ophthalmol Vis Sci 1992; 33: 1384.

11 Sample PA, Taylor JD, Martinez GA, Lusky M, Weinreb $\mathrm{RN}$. Short-wavelength colour visual fields in glaucom suspects at risk. Am $\mathcal{F}$ Ophthalmol 1993; 115: 225-33.

12 Fitzke FW, Poinoosawmy D, Nagasubramanian S, Hitchings RA. Peripheral displacement thresholds in glaucoma and ocular hypertension. In: Heiil A, ed. Perimetry update 1988/9. Amsterdam: Kugler and Ghedini, 399-405.

$13 \mathrm{Wu} X$, Wormald $R$, Fitzke $F$ Poinoosawmy $D$, Nagasubramanian $S$, Hitchings R. Laptop computer perimetry for glaucoma screening. Invest Ophthalmol Vis Sci 1991; 32: 810 .

14 Watkins R, Buckinghan T. Motion perception hyperacuity is abnormal in primary open angle glaucoma and ocula hypertension. Invest Ophthalmol Vis Sci 1991; 32 (suppl) 1103.

15 Bullimore MA, Wood JM, Swenson K. Motion perception in glaucoma. Invest Ophthalmol Vis Sci 1993; 34: 3526-33.

16 Falcao-Reis FM, O'Sullivan F, Spileers W, Hogg C, Arden GB. Macular colour contrast sensitivity in ocular hypertension and glaucoma: evidence for two types of defect. tension and glaucoma: evidence for

17 Yu TC, Falcao-Reis F, Spileers W, Arden GB. Peripheral color contrast. A new screening test for preglaucomatous visual loss. Invest Ophthalmol Vis Sci 1991; 32: 2779-89.

18 Tyler CW. Specific deficits of flicker sensitivity in glaucoma and ocular hypertension. Invest Ophthalmol Vis Sci 1981; 20: 204-12.

19 Weinstein GW, Arden GB, Hitchings RA, Ryan S, Calthorpe CM, Odom JV. The pattern electroretinogram (PERG) in ocular hypertension and glaucoma. Arch Ophthalmol 1988; 106: 923-8.

20 Altman DG, Bland JM. Diagnostic tests 3: receiver operating characteristic plots. BMF 1994; 309: 188.

21 Alexander KR. Psychophysical testing. In: Heckenlively J, Arden GB, eds. Principles and practice of clinical electrophysiology of vision. Chicago: Mosby, 1991: 445-58.

22 Perkins ES. The Bedford Glaucoma Survey. I. Long term follow-up of borderline cases. $\mathrm{Br} \mathcal{F}$ Ophthalmol 1973; 57 179-85.

23 Linner E. Ocular hypertension. I. The clinical course during ten years without therapy. Aqueous humour dynamics. Acta Ophthalmol 1976; 54: 707-20.

24 Kitazawa Y, Horie T, Aoki S, Suzuki M, Nishioka K Untreated ocular hypertension. A long term prospective study. Arch Ophthalmol 1977; 95: 1180-4.

25 Berninger T, Arden GB. The pattern electroretinogram. In: Heckenlively J, Arden GB, eds. Principles and practice of clinical electrophysiology of vision. Chicago: Mosby, 1991: clinical ele $291-300$.

26 Jonas JB, Panda S. Decreased photoreceptor count in human eyes with secondary angle-closure glaucoma Invest Ophthalmol Vis Sci 1992; 33: 2532-6. 